\title{
ADAMTS-12: Functions and Challenges for a Complex Metalloprotease
}

\author{
Yamina Mohamedi ${ }^{1}$, Tania Fontanil ${ }^{1,2}$, Santiago Cal ${ }^{1,3}$, Teresa Cobo ${ }^{4,5 * t}$ and \\ Álvaro J. Obaya ${ }^{3,6 * t}$
}

${ }^{1}$ Departamento de Bioquímica y Biología Molecular, Universidad de Oviedo, Oviedo, Spain, ${ }^{2}$ Departamento de Investigación,
Instituto Ordóñez, Oviedo, Spain, ${ }^{3}$ Instituto Universitario de Oncología, IUOPA, Universidad de Oviedo, Oviedo, Spain,
${ }^{4}$ Departamento de Cirugía y Especialidades Médico-Quirúrgicas, Universidad de Oviedo, Oviedo, Spain, Instituto Asturiano de
Odontología, Oviedo, Spain, ${ }^{6}$ Departamento de Biología Funcional, Área de Fisiología, Universidad de Oviedo, Oviedo, Spain

Nineteen members of the ADAMTS family of secreted zinc metalloproteinases are present in the human degradome. A wide range of different functions are being attributed to these enzymes and the number of their known substrates is considerably increasing in recent

OPEN ACCESS

Edited by:

Salvatore Santamaria, Imperial College London,

United Kingdom

Reviewed by:

Alain Colige,

University of Liège, Belgium

Yi Wu,

Xi'an Jiaotong University, China

*Correspondence:

Teresa Cobo

teresa@iaodontologia.es

Álvaro J. Obaya

ajobaya@uniovi.es

${ }^{t}$ These authors have contributed equally to this work and share last authorship

Specialty section:

This article was submitted to Protein Chemistry and Enzymology, a section of the journal Frontiers in Molecular Biosciences

Received: 27 March 2021 Accepted: 21 April 2021

Published: 30 April 2021

Citation:

Mohamedi Y, Fontanil T, Cal S, Cobo T and Obaya ÁJ (2021) ADAMTS-12:

Functions and Challenges for a

Complex Metalloprotease.

Front. Mol. Biosci. 8:686763.

doi: $10.3389 /$ fmolb.2021.686763 years. ADAMTSs can participate in processes such as fertility, inflammation, arthritis, neuronal and behavioral disorders, as well as cancer. Since its first annotation in 2001, ADAMTS-12 has been described to participate in different processes displayed by members of this family of proteinases. In this sense, ADAMTS-12 performs essential roles in modulation and recovery from inflammatory processes such as colitis, endotoxic sepsis and pancreatitis. ADAMTS-12 has also been involved in cancer development acting either as a tumor suppressor or as a pro-tumoral agent. Furthermore, participation of ADAMTS-12 in arthritis or in neuronal disorders has also been suggested through degradation of components of the extracellular matrix. In addition, ADAMTS-12 proteinase activity can also be modified by interaction with other proteins and thus, can be an alternative way of modulating ADAMTS-12 functions. In this review we revised the most relevant findings about ADAMTS-12 function on the 20th anniversary of its identification.

Keywords: ADAMTS-12, matrix metalloprotease, inflammation, arthritis, schizophrenia, cancer

\section{INTRODUCTION}

Since its first annotation in the human genome 20 years ago (NCBI Gene ID: 81792), ADAMTS-12 has been associated to different biological processes in both physiological and pathological conditions, including development, cancer, osteoarthritis, neurological disorders and inflammation. Following ADAMTS-12 initial identification and characterization, eight more members of the ADAMTS ( $\underline{A}$ Disintegrin And Metalloprotease with ThromboSpondin domains) family of proteinases have been identified, making a total of nineteen the number of members of this family of metalloproteases in mammals (Kelwick et al., 2015). In general, all the members share a complex multidomain architecture. This organization includes a signal peptide, a prodomain, a catalytic domain (proteinase domain characterized by an aspartic residue at the end of the consensus $\mathrm{Zn}^{2+}$-chelating motif HExxHxxGxxHD), a disintegrin-like domain, a central thrombospondin like type-1 (TSP-1) motif, a cysteine-rich domain, a spacer region and a variable number of TSP-1 repeats. Most of the members differ in the number of TSP-1 repeats at the carboxy-end region and 


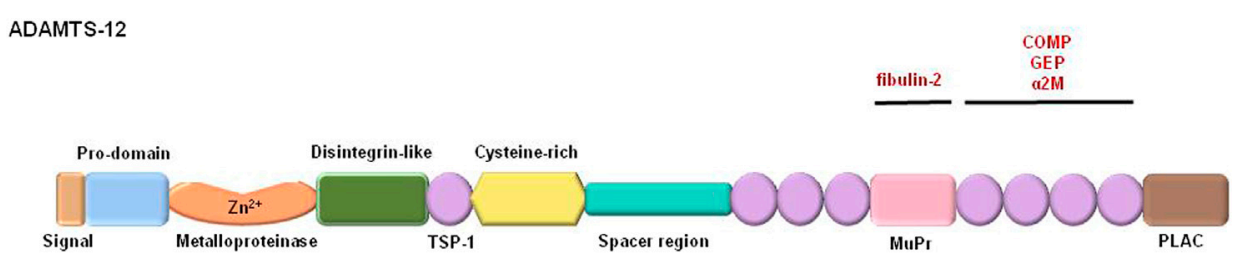

FIGURE 1 | Schematic representation of human ADAMTS-12. In black are shown the main domains of the protein; TSP-1: thrombospondin-like type-1 domain; MuPr: spacer two region. In red are shown proteins known to interact with ADAMTS-12; COMP: cartilage oligomeric matrix protein; GEP: granulin epithelial precursor; a2-macroglobulin.

also by the presence of additional motifs like PLAC, MuPr, cub or GON-1 domains (Porter et al., 2005; Kelwick et al., 2015).

Human ADAMTS12 gene maps at position 5p13 and spans approximately $368.45 \mathrm{~Kb}$ of genomic DNA containing a total of 24 exons (HGNC: 14605). Conceptual translation of human ADAMTS12 cDNA indicates that it encodes for a 1594 amino acid extracellular protein with an estimated molecular weight of $178 \mathrm{kDa}$ (UniProtKB-P58397). ADAMTS12 expression was detected in a variety of tissues like cartilage, lung, kidney, liver, synovium, tendon, skeletal muscle, adipose (Liu, 2009; El Hour et al., 2010; Moncada-Pazos et al., 2012). It is also widely expressed in tumors from different origins and mainly associated with the gastrointestinal tract, suggesting that this enzyme could also participate in the development or progression of this type of tumors (Liu et al., 2006b; Moncada-Pazos et al., 2009; Wang et al., 2011; Fontanil et al., 2014; Liang et al., 2020; Rabadan et al., 2020).

Several studies performed to characterize ADAMTS-12 function suggest its role as a host-protective enzyme with antitumor properties (Llamazares et al., 2007; Moncada-Pazos et al., 2009; El Hour et al., 2010; Wang et al., 2011; Rabadan et al., 2020). ADAMTS-12 also participates in other pathological situations in which a common trail of an inflammatory outburst is present such as the allergen-induced hyperresponsiveness detected in asthma or the extracellular matrix degradation observed in osteoarthritic processes (Liu, 2009; Moncada-Pazos et al., 2012; Nah et al., 2012; Paulissen et al., 2012). Furthermore, in support of previous observations, by different genomic approximations the ADAMTS-12 locus has been associated with several human pathologies like asthma, schizophrenia or predisposition to pediatric stroke (Kurz et al., 2006; Arning et al., 2012; Bespalova et al., 2012). In recent years, genome-wide and bioinformatics analysis techniques have been used with results supporting ADAMTS12 participation in those pathological processes and also in new situations with the benefit of the possibility of being used as a prognosis biomarker ( $\mathrm{Hu}$ et al., 2020; Liang et al., 2020; Zhang et al., 2020). However, besides its participation in pathological situations much more research is needed to identify ADAMTS-12 interacting partners and its substrates in normal or pathological processes. This fact relies in the complex structure of ADAMTS-12 in which multiple domains can be identified and thus, multiple interacting regions are possible for other components of the extracellular matrix (ECM) (Figure 1).

The main processes in which ADAMTS-12 has been proposed to participate will be discussed in the following sections, focusing in the importance of those biological processes in which the associations with ADAMTS-12 have been described.

\section{ADAMTS-12 in Inflammation}

Initial studies aimed to decipher ADAMTS-12 biological function indicate its role as a critical mediator in inflammation processes. Thus, in the human genome screening program ADAMTS12 was identified as a putative asthma associated gene (Kurz et al., 2006). Screening was made in two different populations to identify a chromosome 5-linked asthma or bronchial hyperresponsiveness locus by genotyping 89 single nucleotide polymorphisms (SNPs) in 22 genes and in two different populations (Kurz et al., 2006). Further, in vivo studies were made taking advantage of the availability of the Adamts12 deficient mice and the use of different functional approaches (El Hour et al., 2010; Moncada-Pazos et al., 2012; Paulissen et al., 2012). Two different models of allergic airways disease were used to challenge the Adamts12 deficient mice, ovalbumin (OVA) and house dust mite asthma-induced models. In absence of Adamts12, an elevated eosinophilic inflammation, together with increased levels of mast cells and IL-33, were observed in the alveolar environment suggesting a protective role of this proteinase against this inflammatory process (Paulissen et al., 2012).

Other models of inflammation were performed in the same genetic background and compared to normal mice such as colitis, pancreatitis and endotoxic sepsis. Overall, absence of Adamts12 in mice resulted in a more severe inflammatory phenotype. As mentioned before, a protective role of ADAMTS-12 in inflammatory processes is suggested for these three models which, at the same time, showed some common cellular and molecular patterns. Mice lacking Adamts-12 exhibited an increased tissue damage subsequent to inflammation, accompanied by high levels of pro-inflammatory cytokines like IL-6, IL-11, GCSF, S100A8, S100A9 and also hemopexin, a free heme scavenger protein known for delaying apoptosis of neutrophils (Arruda et al., 2004). Furthermore, neutrophil accumulation has been observed in all the models and in the insulted organs of Adamts12 deficient mice which suggested a required key role of Adamts-12 in neutrophil clearance and subsequent resolution of the inflammatory process (MoncadaPazos et al., 2012).

All together these data suggest that ADAMTS-12 has a protective role or acts as an intermediate factor in the resolution in these inflammatory processes, In addition, 
absence of this proteinase results in a sustain inflammatory phenotype.

\section{ADAMTS-12 in Orteoarthritis}

Osteoarthritis (OA) and rheumatoid arthritis (RA) are believed to be the most prevalent rheumatic diseases, affecting approximately $11 \%$ (10\% OA and 1\% RA) of the world population (Frangos and Maret, 2020). Both, RA and OA are debilitating degenerative diseases affecting articular joints which are characterized by a deregulation in joint or bone homeostasis. Physiological balance is altered and results in the degradation of articular cartilage, alterations in the subchondral bone mass, and also it is also characterized by the presence of localized inflammatory events (Dancevic and McCulloch, 2014; Perez-Garcia et al., 2019a). In addition, this local inflammation is associated with an increase in the production of zinc-dependent matrix metalloproteases which are responsible for the degradation of main components of the extracellular matrix of the cartilaginous tissue (Kevorkian et al., 2004). MMPs and ADAMTSs are known proteases that participate in this process by cleaving some of these components; such is the case of COMP (Cartilage Oligomeric Matrix Protein) and the hyalectan aggrecan (Dickinson et al., 2003; Sandy, 2006). Among them, the association of ADAMTS12 with arthritis and osteoarthritis has been described in several studies along with other members of the ADAMTS family such as ADAMTS-7, and the aggrecanases ADAMTS-4 and ADAMTS-5 (Lin and Liu, 2009; Lin and Liu, 2010).

ADAMTS-12 and ADAMTS-7 share a very similar structure, showing unique domains and thus forming their own subgroup within the ADAMTS family (Kelwick et al., 2015). It is suggested that both enzymes may play key roles in the onset of arthritic events through the ability of cleaving COMP, a pentameric glycoprotein that is part of the extracellular matrix and is used as an indicator of accelerated joint erosion (Liu et al., 2006a; Liu et al., 2006b; Kullich et al., 2007; Liu, 2009). The cleavage of COMP requires an interaction of the EGF-like domain of COMP with the four carboxy-end type 1-thrombospondin repeats found in both ADAMTS-7 and ADAMTS-12 (Guo et al., 2010; Lin and Liu, 2010). The recognition of these binding sites was initially observed by a yeast-two-hybrid genomic screening and subsequently demonstrated by co-immunoprecipitation techniques and in vivo detection (Liu et al., 2006a; Liu et al., 2006b). In fact, ADAMTS-12 participation in arthritic processes has been mainly related to its ability to degrade COMP because fragments generated following its cleavage were found in diseased cartilage, synovial fluid and in serum of patients with knee injuries, osteoarthritis and rheumatoid arthritis (Neidhart et al., 1997; Dickinson et al., 2003; Lin and Liu, 2009). Furthermore, COMP fragments observed in cartilage explants of osteoarthritis patients showed similar size to those identified from in vitro studies (Luan et al., 2008). Elevated levels of ADAMTS-12 in OA and RA, and ADAMTS-7 in RA were detected in the same tissues in which products generated by the cleavage of COMP were identified suggesting that COMP and these enzymes may co-localize (Liu et al., 2006a; Liu et al., 2006b; Luan et al., 2008; Lin and Liu, 2009). In 2008 Luan et al. demonstrated that the COMP fragments found in the cartilage of six patients with OA showed the same size $(110 \mathrm{kDa})$ than those obtained by in vitro degradation of COMP by both proteases. In addition, in cartilage explants the expression of both enzymes increased with the expression of TNF- $\alpha$ and IL- $1 \beta$, therefore favoring COMP cleavage. Moreover, inhibition with antibodies that block either ADAMTS-7 or ADAMTS-12 as well as the use of specific siRNAs kept COMP unprocessed in both, the explants and human chondrocytes (Luan et al., 2008). Additional finding linking ADAMTS-12 with osteoarthritis pathologies came from the identification of ADAMTS12 polymorphisms associated with RA. More specifically three SNPs: rs1364044, intron $\mathrm{C} / \mathrm{T}$; rs10461703, intron $\mathrm{C} / \mathrm{T}$; rs25754, and a missense Thr1495Ile) were identified in this pathology (Nah et al., 2012). All of it together with the fact that ADAMTS12 is one of the genes up-regulated in OA with high levels in cartilage, synovial fluid and serum of arthritic patients supports the idea of ADAMTS12 to be considered as a bona fide gene for the development of antiRA therapies (Kevorkian et al., 2004; Liu et al., 2006b; Nah et al., 2012).

Further confirmation of ADAMTS-12 participation in osteoarthritis processes was recently demonstrated through the employment of an in vivo collagen-induced arthritis (CIA) model by using Adamts12 deficient mice (Wei et al., 2018). Mice lacking Adamts-12 developed accelerated inflammatory arthritis when compared to their control littermates. Bone destruction, increase in synovitis, higher cartilage loss, and increased osteoclastogenesis were the main alterations observed in Adamts12 deficient mice after CIA. This work also pointed out that ADAMTS-12 participation in the arthritic phenotype may be in part caused through proteolytic processing of Connective Tissue Growth factor (CTFG) by ADAMTS-12. The observed data also suggested that CTFG may be a downstream mediator of ADAMTS-12 participation in the inflammatory process (Wei et al., 2018). In this context, ADAMTS-12 is suggested to act as a factor that prevents inflammatory arthritis. In fact, absence of CTGF degradation in Adamts12 deficient mice after IL-1 $\beta$ stimulation led to an increase of pro-inflammatory markers like NOS-2, IL-6 and COX-2 and a reduction of IL-10, a known anti-inflammatory molecule (Wei et al., 2018). Therefore, this data suggests again a protective role for ADAMTS-12 in arthritic events through modulation of the inflammatory landscape.

Interactions between ADAMTSs and different ECM components are of important relevance regarding the participation of these metalloproteases in the regulation of extracellular matrix homeostasis. In fact, degradation of COMP by ADAMTS-7 and ADAMTS-12 is naturally inhibited by the presence of alpha-2-macroglobulin ( $\alpha 2 \mathrm{M})$, which is also an endogenous substrate of both proteases (Luan et al., 2008; Wan et al., 2012). ADAMTS-7 and ADAMTS-12 have also been described to interact and co-localize with Granulin-Epithelin Precursor (GEP), an autocrine growth factor expressed in different pathologies, including RA and OA (Justen et al., 2000). In turn, GEP is also able to block COMP degradation by these two enzymes (Bai et al., 2009a; Guo et al., 2010). GEP binding to either ADAMTS-7 or ADAMTS-12 matches the same domains to which COMP interaction occurs and thus can be 
considered as a competitive inhibitor. GEP and COMP bind to the four C-terminal TSP-I like motifs of both ADAMTSs and it does so in a dose dependent manner. GEP interaction resulted in a GEP proteolytic cleavage that releases several fragments of $\sim 6 \mathrm{kDa}$ known as granulins that can attach to the carboxy-end of both enzymes (Davidson et al., 2004). GEP not only competes directly with COMP for ADAMTS binding but also reduces the levels of both, ADAMTS-7 and ADAMTS-12 through downregulation of TNF- $\alpha$ (Luan et al., 2008).

Cartilage remodeling by uncontrolled activity of chondrocytes is not the unique hallmark in the development of OA since other processes, such as synovial inflammation can also occur (Di Nicola, 2020; Rocha et al., 2021). In this sense, synovial fibroblasts (SF) can also be included as important players in joint destruction in both $\mathrm{RA}$ and $\mathrm{OA}$ with an invasive phenotype. SF, in an inflammation environment, are known to produce and secrete various members of the ADAMTS family which are able to degrade cartilage components such as aggrecan and COMP (Lefevre et al., 2015; van Nie et al., 2020). Moreover, inflammatory cytokines like IL-1 $\beta$ as well as fibronectin fragments can induce an increase of ADAMTS-12 expression, both at the mRNA and protein levels, in SF from OA patients when compared with healthy synovial fibroblasts and therefore increase the degradation of COMP. Something similar occurs with ADAMTS-7 with the difference that in this case it occurred in both types of fibroblasts (Perez-Garcia et al., 2016).

Mechanism by which IL-1 $\beta$ mediates ADAMTS-7 and ADAMTS-12 expression in SF was recently described and involves two different pathways, Runx 2 and $\mathrm{Wnt} / \beta$-catenin. Presence of specific inhibitors for both pathways in SF cocultured with cartilage explants from patients with OA were used to demonstrate that ADAMTS-7 expression depends on the Wnt/ $\beta$-catenin pathway, whereas ADAMTS-12 depends on the ERK pathway and ultimately in the activation of Runx2. It was also shown that IL- $1 \beta$ can induce expression of ADAMTS-7 and ADAMTS-12 which led to COMP degradation. However, while IL- $1 \beta$ can induce both ADAMTSs, the presence of fibronectin fragments was only able to induce ADAMTS-12 expression and the consequent COMP degradation (PerezGarcia et al., 2019b).

In summary, involvement of ADAMTS-12 in arthritic processes has been demonstrated by its ability of degrading essential components such as COMP. Production of inflammatory mediators (for example IL-1 $\beta$ ) induces ADAMTS-12 expression which through COMP cleavage releases other component of the extracellular matrix with proinflammatory effects. As a result, all these molecular interactions generate a positive feedback loop that increases joint damage which is a characteristic observed in osteoarthritic processes (Liu et al., 2006b; Perez-Garcia et al., 2016; PerezGarcia et al., 2019b). In addition, ADAMTS-12 seems to be also responsible for limiting inflammatory arthritis by modulating pro- and anti-inflammatory molecules. Degradation of other ECM molecules like CTFG can be considered part of an important mechanism for the resolution of the arthritic inflammatory phenotype.

\section{ADAMTS-12 in Chondrogenesis}

ADAMTS-12 plays an important role in the regulation of chondrogenesis and cartilage development. Levels of ADAMTS-12 have been detected strongly upregulated during chondrogenesis and also in proliferating and hypertrophic chondrocytes (Jin et al., 2007; Bai et al., 2009a; Bai et al., 2009b; Jahangir et al., 2020). ADAMTS-12 role in chondrogenesis is related to an important chondrogenic regulator, Parathyroid Hormone-Related Peptide (PTHrP) (Rajagopal et al., 2020). A feedback loop beween PTHrP and ADAMTS-12 has been observed in terms that ADAMTS-12 induces PTHrP and ADAMTS-12 is barely detectable in PTHrP -/- growth plate chondrocytes since PTHrP, at the same time induces ADAMTS-12 expression. The effects caused by these two molecules result in the inhibition of chondrocyte differentiation through the inhibition of genes such as type II collagen (Col II) or Sox9, markers of chondrocyte differentiation (Bai et al., 2009b).

On the other hand, genetic studies in a family of patients with autosomal-dominant Brachydactyly Type E disorder had led to the identification of a $(8 ; 12)(\mathrm{q} 13 ; \mathrm{p} 11.2)$ translocation that affected the PTHLH gene which encodes for PTHrP (Maass et al., 2010). Since this translocation affects PTHrP locus, it also has consequences on its downstream targets, ADAMTS-7 and ADAMTS-12, therefore affecting normal chondrogenic differentiation.

Another molecule that regulates the activity of ADAMTS-12 in chondrogenesis is the transcription factor c-Maf, a member of the Maf family of basic ZIP transcription factors (bZIP). Among other functions, c-Maf is known for its involvement in chondrogenesis (Hong et al., 2011; Hong et al., 2013). Thus, expression of c-Maf is maximal in hypertrophic chondrocytes during embryonic development and postnatal growth. On the other hand, ADAMTS-12 expression increases during in vitro chondrogenesis of human mesenchymal stem cells as well as during mouse embryonic limb development (Bai et al., 2009b; Hong et al., 2011), A "Maf recognition element" (MARE) has been identified in the ADAMTS12 proximal promoter where, through the use of luciferase reporter assays, c-Maf binding has been demonstrated to induce ADAMTS-12 expression. Therefore, c-Maf effects on differentiation and hypertrophy of chondrocytes might be in part through up regulation of ADAMTS-12 (Hong et al., 2013).

\section{ADAMTS-12 in Degenerative Intervertebral Disc}

Between each vertebral body of the spine are pads of fibrocartilage-based structures that provide support, flexibility, and a better load distribution known as intervertebral discs. Each of them also contains a soft tissue within the interior which is known as nucleus pulposus (Donnally et al., 2021). As happens in arthritic processes and other inflammatory disorders, the intervertebral discs can be subjected to degradation by molecules capable of processing extracellular matrix components as is the case of MMPs and ADAMTSs (Le Maitre et al., 2007). In this regard, high levels of ADAMTS-7 
and ADAMTS-12 have been observed in nuclei pulposus extracted from rats in which disc degeneration was generated through the use of an external compression device ( $\mathrm{Yu}$ and $\mathrm{Zhu}$, 2012). These results were accompanied by an increase in COMP fragments (Liu et al., 2006b; Yu and Zhu, 2012). In addition, levels of both proteases are increased in endplate cells isolated from patients with IVD suggesting their participation in this pathological process through degradation of the ECM components (Zhang et al., 2012).

All of these data would indicate that ADAMTS-7 and ADAMTS-12 contribute to intervertebral disc degeneration through a molecular mechanism similar to that described for the osteoarthritic disorders ( $\mathrm{Yu}$ and Zhu, 2012; Zhang et al., 2012).

\section{ADAMTS-12 in Ossification and Tendon Degeneration}

Presence of higher levels of ADAMTS-7 and ADAMTS-12 at mRNA and protein levels in degenerative IVD when compared with normal IVD seems to be associated with low levels of Col II, Sox 9 and Col X, known chondrogenesis marker genes (Zhang et al., 2012). ADAMTS-7 and ADAMTS-12 are associated to cartilage destruction and endochondral ossification (Lin and Liu, 2009). Adamts7 and Adamts12 deficient mice are viable with no apparent phenotype. Adamts7 knockout was initially used to validate the association of Adamts-7 with atherogenesis while Adamts12 knockout served as a model to describe Adamts-12 antitumoral function (El Hour et al., 2010; Bauer et al., 2015). Both proteases share an important structural homology and have shown mutual compensation in hindlimb tendons, with upregulation of one of them when the other one is absent (Mead et al., 2018). Double knockout of both ADAMTSs has been generated to prove their participation in skeletal development and more specifically, in heterotopic ossification (HO) of tendons and ligaments (Mead et al., 2018). Furthermore, these mice developed normally, with no skeletal anomalies till the age of 4 months when $\mathrm{HO}$ was identified within the quadriceps tendon, Achilles tendon and menisci (Mead et al., 2018). Extension of the study to human pathologies showed that reduction of ADAMTS-7 and ADAMTS-12 immunostaining was evident in human degenerative biceps tendons although only ADAMTS7 mRNA was reduced significantly (Mead et al., 2018).

Participation of ADAMTS-12 and ADAMTS-7 in ECM remodeling in musculoskeletal tissues is important for homeostasis maintenance. The above studies indicate that both proteases are basally expressed in bone, cartilage, synovium, ligament and tendons and thus being implicated in normal turnover of these tissues. Alterations on their expression could lead to the development of different pathologies like arthritis, IVD degeneration and ossification. Furthermore, the structural similarity of ADAMTS-7 and ADAMTS-12 can generate compensatory mechanisms between these proteases in those body compartments in which both are normally expressed.

\section{ADAMTS-12 in Neurological Disorders}

In 2012, Bespalova et al. (Bespalova et al., 2012) identified a schizophrenia susceptibility locus on chromosome $5 \mathrm{p} 13$. This region contains the ADAMTS12 gene and to study its potential involvement in the disease, a mutation analysis was performed in Puerto Rican patients of Spanish descent. That analysis revealed that one intronic variant and two SNP haplotypes were closely linked to the susceptibility of developing schizophrenia. Previously, sequence variants in the ADAMTS12 gene had also been associated to bipolar disorder and narcolepsy using genome-wide association studies (GWAS) in Japanese population (Hattori et al., 2009; Koike et al., 2009). GWAS technique has also allowed to include ADAMTS12 gene as functionally linked to Alzheimer's disease (Wang et al., 2015). Taking together, those studies showed clear evidence that genetic variations of the ADAMTS12 gene could underlie mental health disorders.

Also in 2012, a study was published highlighting the association between the ADAMTS12 gene and pediatric stroke using GWAS (Arning et al., 2012). Further evidence that ADAMTS12 could display a role in pediatric stroke was obtained through the sequencing and fine mapping of ADAMTS12 gene variants (Witten et al., 2020). Similar type of analysis was performed to determine that an ADAMTS-12 variant could offer a protective effect against cerebral aneurysm (Arning et al., 2016). These results suggest that ADAMTS12 could be considered a very promising factor to analyse in relation to its capacity to associate to other ECM components of the CNS. Moreover, ADAMTS-12 might also potentially induce modifications in ECM components through its catalytic activity. In consequence, an altered ADAMTS-12 metalloprotease could lead to a disrupted ECM. These studies also open the possibility of ADAMTS- 12 could be a potential diagnostic and prognostic biomarker in cerebrovascular diseases. However, it would be necessary to explore the functional mechanisms associated to ADAMTS-12 activities in neurological system.

In order to shed light about the functional role of ADAMTS12 in the central nervous system, we recently studied its expression in different areas of mouse brain at different developmental stages (Fontanil et al., 2019). Expression was detected at different levels in embryonic stages, but was very low or undetected in the postnatal or adult stages analyzed. As a potential substrate of ADAMTS-12 in brain, neurocan expression was also evaluated with the finding that this hyalectan displayed a similar expression pattern as that shown by the metalloprotease. Gene expression analysis indicates that both, neurocan and ADAMTS12, can be detected in the olfactory bulb in the brain of adult mice. Adamts12 knockout mice develop normally with no apparent behavior deficiencies or CNS alterations. However, the absence of Adamts-12 results in neurocan accumulation in specific areas of the CNS such as olfactory bulb, hypothalamus and spinal ganglia of new born mouse (Fontanil et al., 2019). This accumulation potentially excludes neurocan processing by other proteases. Neurocan cleavage not only by ADAMTS-12 but also by other ADAMTS family members could be crucial for normal maintenance of brain function (Tauchi et al., 2012; Gottschall and Howell, 2015; Fontanil et al., 2021). In this regard, neurocan 
has been related with important functions in brain development. For instance, it has been recently reported that neurocan contributes to the perineuronal nets formation during postnatal development in mouse (Schmidt et al., 2020). Moreover, neurocan levels increase during traumatic brain injury (Asher et al., 2000), which could contributed to generate a three-dimensional net together other ECM components for cells in order to repair the damage (Fontanil et al., 2019). It is also noteworthy that gene encoding neurocan, NCAN, has been associated with mental health diseases (Avram et al., 2014) including schizophrenia and bipolar disorder (Muhleisen et al., 2012; Oruc et al., 2012; Wang et al., 2016) or related with the etiology of mania (Miro et al., 2012). Different studies have connected the rs1064395 SNP within NCAN gene with those disorders (Raum et al., 2015; Wang et al., 2016). It has been recently shown that the NCAN rs1064395 A allele is linked to lower hippocampus-dependent memory function (Assmann et al., 2020). Furthermore, that SNP influences the expression of the neighboring HAPLN4 gene (Assmann et al., 2020). This gene codes for the ECM link protein Hapln4/Bral2, which show a strong expression in human cortex (Bekku et al., 2003; Spicer et al., 2003). These studies point to the strong influence of this genetic variant of the NCAN gene on the ECM composition, highlighting the influence of neurocan in both normal and pathological conditions of the CNS. In addition, a growing body of evidence suggests that ADAMTS-12 could influence brain function through different mechanisms, including its functional relationship with neurocan.

\section{ADAMTS-12 in Cancer}

ADAMTS-12, like many other members of the ADAMTS family, may have a pro- or anti-tumor role in various types of cancers. Initially, ADAMTS-12 was described as a tumor suppressor protein, as the exogenous expression of ADAMTS-12 in Madin-Darby Canine Kidney (MDCK) cells prevents the phenotypic changes associated with renal carcinogenesis caused by the growth factor of hepatocytes (HGF). This effect is due to the thrombospondin motifs that this enzyme exhibits at the C-terminal end, and which block the Ras-MAPK signalling pathway (Llamazares et al., 2007). MDCK cells expressing a truncated form of ADAMTS-12 lacking the TSP-1 repeats also showed tumorigenic effects compatible with those previously described. ADAMTS-12 overexpression in A549 lung adenocarcinoma cells inhibits subcutaneous tumor formation in immunodeficient SCID mice in comparison with animals injected with A549 parental cells. In addition, ADAMTS12 gene is epigenetically silenced by hypermethylation of its promoter in tumors of different sources, which reinforced the hypothesis of ADAMTS12 as a tumor suppressor (MoncadaPazos et al., 2009). Furthermore, in colon tumors there is a dual regulation of ADAMTS-12 expression: first, an epigenetic inactivation in colon cancer cells due to high levels of methylation of ADAMTS12 promoter. And second, an overexpression of ADAMTS-12 by the fibroblasts surrounding neoplastic cells was detected, which suggest a protective stromal response to reduce tumor progression. Other studies support the protective role of ADAMTS-12 in colorectal cancer (Wang et al.,
2011; Zheng et al., 2019). However, it has also been described that ADAMTS-12 can promote the migration and proliferative properties of HCT116 colorectal carcinoma cells by activating the Wnt/ $\beta$-catenin signaling pathway (Li et al., 2020a). On the other hand, the phenotypic analysis of the Adamts 12 deficient mouse indicated that this protein plays a protective role against angiogenesis and tumor growth. In fact, mice lacking an active Adamts-12 showed high levels of vascularization and tumor invasion after malignant keratinocyte transplantation (El Hour et al., 2010). These mice also showed a greater number of lung tumors when compared with their normal littermates after administration of the carcinogen urethane. In addition, in vitro studies using M-38 lung carcinoma cells revealed an increase in their proliferative and invasive potential after being depleted of ADAMTS-12 using RNA interference techniques. Protective role of ADAMTS-12 in lung cancer was also supported through the employment of bioinformatic approaches. This analysis would indicate that patients with lung adenocarcinoma in which truncating mutations in the ADAMTS12 gene were detected, would have a worse prognosis than those with the unaltered gene (Rabadan et al., 2020).

A protective role of ADAMTS-12 has also been found in breast cancer through its association with fibulin-2 (Fontanil et al., 2014). In this case, fibulin-2/ADAMTS-12 interaction was demonstrated after yeast-two-hybrid screening and inmunoprecipitation studies. ADAMTS-12 exogenously expressed in breast cancer cells increases the formation of subcutaneous tumors in immunodeficient SCID mice, and the capacity of migration, invasion, and mammosphere formation of these cancer cells, which can be associated to a pro-tumoral effect. However, presence of fibulin-2 and ADAMTS-12 is able to block those properties suggesting a protective role induced by that interaction (Fontanil et al., 2014). In fact, fibulin-2 can be degraded by ADAMTS-4 and ADAMTS-5 under the same experimental conditions, which results in an increase of the tumoral properties of breast cancer cells (Fontanil et al., 2017). The interaction of ADAMTS-12 with fibulin-2 results in a blockade of the proteolytic degradation of fibulin-2 by ADAMTS4 and ADAMTS-5 and therefore, inhibition of the tumoral properties of breast cancer cells (Fontanil et al., 2017).

In a stark contrast, ADAMTS-12 has also been associated to protumoral effects. Thus, it has been shown that the overexpression of ADAMTS-12 in human trophoblastic cells enhances their invasive phenotype through regulating the expression and function of the integrin $\alpha_{\mathrm{v}} \beta_{3}$ (Beristain et al., 2011). In addition, ADAMTS- 12 plays a pro-tumoral role in breast cancer, by increasing the formation of subcutaneous tumors in immunodeficient SCID mice, and the capacity for migration, invasion, and mammosphere formation in breast cancer tumor cells. However, when ADAMTS-12 interacts with fibulin-2, these effects are reversed, promoting an antitumor role (Fontanil et al., 2014). Other studies seem to suggest the tumorigenic function of this protein since the gene encoding for ADAMTS12 has been identified as one of the genes overexpressed in ovarian cancer patients with intestinal metastases (Mariani et al., 2019), in patients with metastatic 
renal carcinoma (Ho et al., 2017), gastric carcinoma with poor prognosis (Liang et al., 2020) and esophageal squamous cell carcinoma (Li et al., 2020b).

With the previous data on mind, analysis and correlation studies are of key importance to decipher the precise role of ADAMTS-12 in cancer development. In fact, ADAMTS-12 seems to be preferentially associated with cancer associated fibroblasts (CAFs) and immune cells (macrophages) in colorectal cancer (Moncada-Pazos et al., 2009; Wang et al., 2011). ADAMTS-12 has been specifically detected in activated fibroblasts in the proximity of colon cancer cells. Furthermore, co-cultured fibroblasts are able to induce colon cancer cells death by apoptotic mechanisms (Moncada-Pazos et al., 2009). In the urethane-induced lung cancer model, ADAMTS-12 is present in cells surrounding the highly proliferative cells within the tumor (Rabadan et al., 2020). In breast cancer, ADAMTS-12 expression is localized preferentially surrounding the tumoral tissue and high expression is correlated with good prognosis of these patients (Fontanil et al., 2014). On the other hand, expression analysis in gastric cancer has revealed high levels of ADAMTS-12 in tumoral samples when compared to adjacent tissue and a correlation with poor prognosis (Liang et al., 2020). In this sense, the availability of gene expression databanks can be a great tool to analyze gene participation in cancer. However, care must be taken in order to define gene groups when analyzing complex samples from tumors in which types of cells from different origin can be found (fibroblasts, cancer cells, endothelial, muscle cells and immune cells among others). In addition, ADAMTS-12 participation in the resolution of inflammatory processes should also be taking into account in local cancer development. Inflammatory molecules and the immune response have been described to be drivers and to potentiate cancer development (Suarez-Carmona et al., 2017; Gupta et al., 2018). Pro-inflammatory cytokines are also potent chemoattractants for immune cells that, consequently, increase the local inflammatory environment. ADAMTS-12 participation in modulation and resolution of inflammatory processes might as well be part of its antitumoral effect. In fact, ADAMTS-12 can reduce levels of inflammatory molecules by degradation of CTFG and, at the same time it is also able to induce neutrophil clearance by inducing cell apoptosis (Moncada-Pazos et al., 2012; Wei et al., 2018).

In conclusion, ADAMTS-12 can be related to act as a tumoral suppressor and also to act as a protumoral agent. In this sense, ADAMTS-12 function may depend on several factors, type of cells in which it is expressed (cancer cells, inmune or stromal cells), the appearance of ADAMTS-12 mutants (point mutations, truncated forms, protein processing) as well as the interactions with other components of the ECM.

\section{ADAMTS-12 in Fertility Disorders}

The initial phenotypic analysis of Adamts12 deficient mice showed no alterations in reproduction, with normal gestation periods and fertility. However, the expression of Adamts12 in wild type mice was detected in tissues such as the mammary glands, uterus and ovary, but not in testes (El Hour et al., 2010). In addition, ADAMTS-12 plays a role in angiogenesis and inflammation, which may imply that this protein can be involved in processes such as the formation of the placenta and problems associated with pregnancy as is the case of preeclampsia. Moreover, ADAMTS-12 affects the invasion of trophoblastic cells and their adhesion to components of the extracellular matrix by regulating the expression of the integrin $\alpha_{\mathrm{v}} \beta_{3}$ (Beristain et al., 2011). In fact, high levels of ADAMTS-12 are detected in placenta during the first three months of pregnancy, which highlights the important role that this protein plays in the implantation of this organ. Regarding the role of ADAMTS-12 in pre-eclampsia, data are controversial, as a decrease in ADAMTS-12 levels have been detected in women with pre-eclampsia (Eda Gokdemir et al., 2016). However, other studies found an increase in ADAMTS-12 levels in placenta (but not in maternal blood or umbilical cord) in patients with preeclampsia (Namli Kalem et al., 2018). Finally, other studies did not detect differences in these groups (Daglar et al., 2016; Kirbas et al., 2016; Oztas et al., 2016) but a decrease in levels of ADAMTS-12 in placenta during pregnancy-associated cholestasis (Oztas et al., 2016). Recently, it has also been reported that women with spontaneous preterm birth present hypomethylation of ADAMTS12 promotor in the placenta tissue (Mani et al., 2019).

\section{Perspectives in ADAMTS-12 Functional Biology}

During the past two decades several studies have associated ADAMTS-12 with different pathologies as well as being part of physiological processes (Figure 2). ADAMTS-12 has been initially identified as a member of the wide family of $\mathrm{Zn}^{2+}$. metalloproteases. However, ADAMTS-12 needs to be considered much more than a simple proteinase due to its complex structure that contains multiple different domains able to interact with other components of the ECM. These interactions not only could be important for the proteolytic activity of the enzyme but can also sequester different factors, bring to proximity components of the extracellular matrix or trigger cellular responses. For instance, it is known that ADAMTS-12 interacts with other ECM components like fibulin-2, CTFG, COMP, GEP, $\alpha 2 \mathrm{M}$ and can also interact with growth factors such as VEGF (Liu et al., 2006b; Llamazares et al., 2007; Luan et al., 2008; Wan et al., 2012; Fontanil et al., 2014; Wei et al., 2018). In addition, ADAMTS-12 is known to be processed which generates two fragments, one with the proteolytic domain (amino-terminal end) and the other one containing different anchoring domains (mainly TSP-1 repeats) (carboxy-terminal end) which, in turn, can generate the appearance of different activities (Llamazares et al., 2007; Beristain et al., 2011; Wei et al., 2018). All these facts could be relevant to ADAMTS-12 biological function since it is generally accepted that C-terminal domains of metalloproteinases are often important for determining substrate specificity (Martel-Pelletier et al., 2001).

ADAMTS-12 participates in different processes related to an inflammatory response such as asthma, colitis, sepsis, pancreatitis and osteoarthritis. In this sense, mechanisms about ADAMTS-12 participation and regulation of these inflammatory events are 


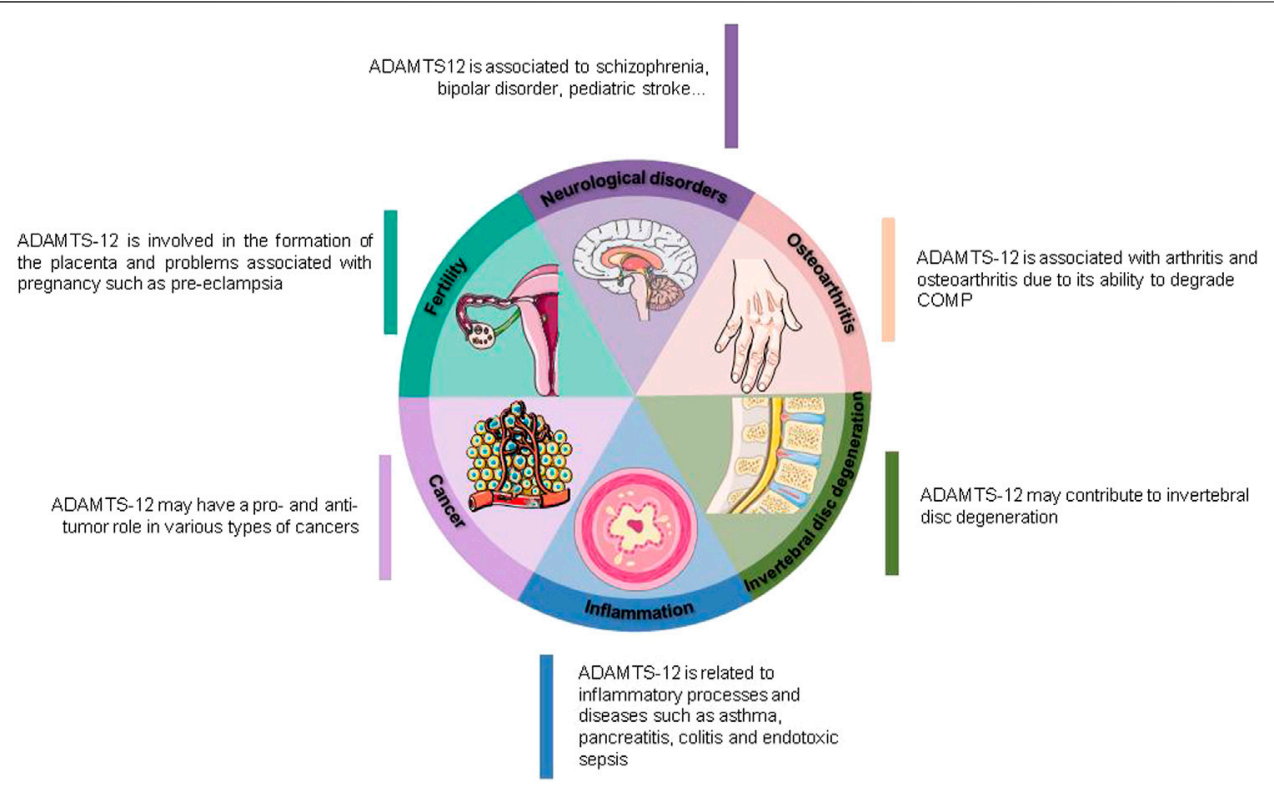

FIGURE 2 | ADAMTS-12 biological implications.

known to be through interaction and degradation of different components of the ECM. It is interesting to note that this enzyme is able to cleave key proteins of cartilage like COMP and, at the same time, degrade other components like CTGF which seems to be required for reducing the inflammatory response (Liu et al., 2006b; Wei et al., 2018). In addition, ADAMTS-12 may have other anti-inflammatory actions by blocking the appearance of elevated levels of pro-inflammatory molecules as well as a role in neutrophil clearance (Moncada-Pazos et al., 2012). Therefore, ADAMTS- 12 can be considered an important player in mediating and resolving the inflammatory response and, its absence may result in pathologies known to be due in part to a not well resolved inflammatory process like can be the case of schizophrenia (Asher et al., 2000; Fontanil et al., 2019).

In summary, ADAMTS-12 can be a key player in the control and regulation of different biological processes, both normal and pathological. It is clearly associated with inflammatory processes but also seems to have an important role in cancer, neurological disorders as well as being related with fertility disorders. Further studies on patients with infrequent anomalies as well as those that have been treated with radioisotopes have found that ADAMTS12 can also be part of the biological mechanism of Freiberg-s infraction, brachydactyly A1B as well as salivary gland damage (Koca et al., 2013; Mysliwiec et al., 2015; Sadic et al., 2016).

As mention in this review, ADAMTS-12 has a complex architecture which can support different activities and hence, be associated to different physiological a pathological processes. The availability of mice deficient in this protease is a useful tool in order to demonstrate its participation in those processes through the use of different experimental models. Furthermore, bioinformatics applications are also being widely used to make associations of ADAMTS-12 with several human diseases. However, more bench work is needed to solve ADAMTS-12 biological function, its interactions with other components of the ECM, the existence of specific substrates and the implications of the different parts of the molecule in regulating those functions. It would be also crucial to identify new interacting partners of this complex metalloprotease within the ECM. This would open the possibility to clarify controversial aspects about the functional relevance of ADAMTS-12.

\section{AUTHOR CONTRIBUTIONS}

Conceptualization, TC and ÁO; data collection, YM, TF, and SC; manuscript editing, SC, TC and ÁO; figure design, YM, TF. All authors have read and agreed to the published version of the manuscript.

\section{FUNDING}

This research was funded by RTI 2018-099756B-100 from the Ministerio Español de Ciencia, Innovación y Universidades and by the Instituto Asturiano de Odolontología, IAO.

\section{ACKNOWLEDGMENTS}

Authors thank Juan Cobo and José A. Vega (Universidad de Oviedo) for critical reading of the manuscript. 


\section{REFERENCES}

Arning, A., Hiersche, M., Witten, A., Kurlemann, G., Kurnik, K., Manner, D., et al. (2012). A Genome-wide Association Study Identifies a Gene Network of ADAMTS Genes in the Predisposition to Pediatric Stroke. Blood 120 (26), 5231-5236. doi:10.1182/blood-2012-07-442038

Arning, A., Jeibmann, A., Kohnemann, S., Brokinkel, B., Ewelt, C., Berger, K., et al. (2016). ADAMTS Genes and the Risk of Cerebral Aneurysm. J. Neurosurg. 125 (2), 269-274. doi:10.3171/2015.7.JNS154

Arruda, M. A., Rossi, A. G., de Freitas, M. S., Barja-Fidalgo, C., and Graca-Souza, A. V. (2004). Heme Inhibits Human Neutrophil Apoptosis: Involvement of Phosphoinositide 3-kinase, MAPK, and NF-kappaB. J. Immunol. 173 (3), 2023-2030. doi:10.4049/jimmunol.173.3.2023

Asher, R. A., Morgenstern, D. A., Fidler, P. S., Adcock, K. H., Oohira, A., Braistead, J. E., et al. (2000). Neurocan Is Upregulated in Injured Brain and in CytokineTreated Astrocytes. J. Neurosci. 20 (7), 2427-2438. doi:10.1523/JNEUROSCI. 20-07-02427.2000

Assmann, A., Richter, A., Schutze, H., Soch, J., Barman, A., Behnisch, G., et al. (2020). Neurocan Genome-wide Psychiatric Risk Variant Affects Explicit Memory Performance and Hippocampal Function in Healthy Humans. Eur. J. Neurosci., 1-18. doi:10.1111/ejn.14872

Avram, S., Shaposhnikov, S., Buiu, C., and Mernea, M. (2014). Chondroitin Sulfate Proteoglycans: Structure-Function Relationship with Implication in Neural Development and Brain Disorders. Biomed. Res. Int. 2014, 642798. doi:10.1155/ 2014/642798

Bai, X. H., Wang, D. W., Kong, L., Zhang, Y., Luan, Y., Kobayashi, T., et al. (2009a). ADAMTS-7, a Direct Target of PTHrP, Adversely Regulates Endochondral Bone Growth by Associating with and Inactivating GEP Growth Factor. Mol. Cel. Biol. 29 (15), 4201-4219. doi:10.1128/MCB.00056-09

Bai, X. H., Wang, D. W., Luan, Y., Yu, X. P., and Liu, C. J. (2009b). Regulation of Chondrocyte Differentiation by ADAMTS-12 Metalloproteinase Depends on its Enzymatic Activity. Cell Mol. Life Sci. 66 (4), 667-680. doi:10.1007/s00018008-8633-x

Bauer, R. C., Tohyama, J., Cui, J., Cheng, L., Yang, J., Zhang, X., et al. (2015). Knockout of Adamts7, a Novel Coronary Artery Disease Locus in Humans, Reduces Atherosclerosis in Mice. Circulation. 131 (13), 1202-1213. doi:10. 1161/CIRCULATIONAHA.114.012669

Bekku, Y., Su, W. D., Hirakawa, S., Fassler, R., Ohtsuka, A., Kang, J. S., et al. (2003). Molecular Cloning of Bral2, a Novel Brain-specific Link Protein, and Immunohistochemical Colocalization with Brevican in Perineuronal Nets. Mol. Cel Neurosci. 24 (1), 148-159. doi:10.1016/s1044-7431(03)00133-7

Beristain, A. G., Zhu, H., and Leung, P. C. (2011). Regulated Expression of ADAMTS-12 in Human Trophoblastic Cells: a Role for ADAMTS-12 in Epithelial Cell Invasion? PLoS One 6 (4), e18473. doi:10.1371/journal.pone. 0018473

Bespalova, I. N., Angelo, G. W., Ritter, B. P., Hunter, J., Reyes-Rabanillo, M. L., Siever, L. J., et al. (2012). Genetic Variations in the ADAMTS12 Gene Are Associated with Schizophrenia in Puerto Rican Patients of Spanish Descent. Neuromolecular Med. 14 (1), 53-64. doi:10.1007/s12017-012-8169-y

Daglar, K., Kirbas, A., Timur, H., Ozturk Inal, Z., and Danisman, N. (2016). Placental Levels of Total Oxidative and Anti-oxidative Status, ADAMTS-12 and Decorin in Early- and Late-Onset Severe Preeclampsia. J. Matern. Fetal Neonatal. Med. 29 (24), 4059-4064. doi:10.3109/14767058.2016.1154942

Dancevic, C. M., and McCulloch, D. R. (2014). Current and Emerging Therapeutic Strategies for Preventing Inflammation and Aggrecanase-Mediated Cartilage Destruction in Arthritis. Arthritis Res. Ther. 16 (5), 429. doi:10.1186/s13075014-0429-9

Davidson, B., Alejandro, E., Florenes, V. A., Goderstad, J. M., Risberg, B., Kristensen, G. B., et al. (2004). Granulin-epithelin Precursor Is a Novel Prognostic Marker in Epithelial Ovarian Carcinoma. Cancer 100 (10), 2139-2147. doi:10.1002/cncr.20219

Di Nicola, V. (2020). Degenerative Osteoarthritis a Reversible Chronic Disease. Regen. Ther. 15, 149-160. doi:10.1016/j.reth.2020.07.007

Dickinson, S. C., Vankemmelbeke, M. N., Buttle, D. J., Rosenberg, K., Heinegard, D., and Hollander, A. P. (2003). Cleavage of Cartilage Oligomeric Matrix Protein (Thrombospondin-5) by Matrix Metalloproteinases and a Disintegrin and Metalloproteinase with Thrombospondin Motifs. Matrix Biol. 22 (3), 267-278. doi:10.1016/s0945-053x(03)00034-9

Donnally, I. C., Hanna, A., and Varacallo, M. (2021). StatPearls.Lumbar Degenerative Disk Disease(Treasure Island (FL)).

Eda Gokdemir, I., Ozdegirmenci, O., Elmas, B., Sarikaya, E., Tokmak, A., Kazanci, F. H., et al. (2016). Evaluation of ADAMTS12, ADAMTS16, ADAMTS18 and IL-33 Serum Levels in Pre-eclampsia. J. Matern. Fetal Neonatal. Med. 29 (15), 2451-2456. doi:10.3109/14767058.2015.1087497

El Hour, M., Moncada-Pazos, A., Blacher, S., Masset, A., Cal, S., Berndt, S., et al. (2010). Higher Sensitivity of Adamts12-Deficient Mice to Tumor Growth and Angiogenesis. Oncogene 29 (20), 3025-3032. doi:10.1038/onc.2010.49

Fontanil, T., Rua, S., Llamazares, M., Moncada-Pazos, A., Quiros, P. M., GarciaSuarez, O., et al. (2014). Interaction between the ADAMTS-12 Metalloprotease and Fibulin-2 Induces Tumor-Suppressive Effects in Breast Cancer Cells. Oncotarget 5 (5), 1253-1264. doi:10.18632/oncotarget.1690

Fontanil, T., Alvarez-Teijeiro, S., Villaronga, M. A., Mohamedi, Y., Solares, L., Moncada-Pazos, A., et al. (2017). Cleavage of Fibulin-2 by the Aggrecanases ADAMTS-4 and ADAMTS-5 Contributes to the Tumorigenic Potential of Breast Cancer Cells. Oncotarget 8 (8), 13716-13729. doi:10.18632/oncotarget. 14627

Fontanil, T., Mohamedi, Y., Moncada-Pazos, A., Cobo, T., Vega, J. A., Cobo, J. L., et al. (2019). Neurocan Is a New Substrate for the ADAMTS12 Metalloprotease: Potential Implications in Neuropathies. Cell Physiol. Biochem. 52 (5), 1003-1016. doi:10.33594/000000069

Fontanil, T., Mohamedi, Y., Espina-Casado, J., Obaya, A. J., Cobo, T., and Cal, S. (2021). Hyalectanase Activities by the ADAMTS Metalloproteases. Int. J. Mol. Sci. 22 (6). 2988. doi:10.3390/ijms 22062988

Frangos, T., and Maret, W. (2020). Zinc and Cadmium in the Aetiology and Pathogenesis of Osteoarthritis and Rheumatoid Arthritis. Nutrients 13 (1), 53. doi:10.3390/nu13010053

Gottschall, P. E., and Howell, M. D. (2015). ADAMTS Expression and Function in Central Nervous System Injury and Disorders. Matrix Biol. 44-46, 70-76. doi:10.1016/j.matbio.2015.01.014

Guo, F., Lai, Y., Tian, Q., Lin, E. A., Kong, L., and Liu, C. (2010). Granulin-epithelin Precursor Binds Directly to ADAMTS-7 and ADAMTS-12 and Inhibits Their Degradation of Cartilage Oligomeric Matrix Protein. Arthritis Rheum. 62 (7), 2023-2036. doi:10.1002/art.27491

Gupta, S. C., Kunnumakkara, A. B., Aggarwal, S., and Aggarwal, B. B. (2018). Inflammation, a Double-Edge Sword for Cancer and Other Age-Related Diseases. Front. Immunol. 9, 2160. doi:10.3389/fimmu.2018.02160

Hattori, E., Toyota, T., Ishitsuka, Y., Iwayama, Y., Yamada, K., Ujike, H., et al. (2009). Preliminary Genome-wide Association Study of Bipolar Disorder in the Japanese Population. Am. J. Med. Genet. B Neuropsychiatr. Genet. 150B (8), 1110-1117. doi:10.1002/ajmg.b.30941

Ho, T. H., Serie, D. J., Parasramka, M., Cheville, J. C., Bot, B. M., Tan, W., et al. (2017). Differential Gene Expression Profiling of Matched Primary Renal Cell Carcinoma and Metastases Reveals Upregulation of Extracellular Matrix Genes. Ann. Oncol. 28 (3), 604-610. doi:10.1093/annonc/mdw652

Hong, E., Di Cesare, P. E., and Haudenschild, D. R. (2011). Role of C-Maf in Chondrocyte Differentiation: A Review. Cartilage 2 (1), 27-35. doi:10.1177/ 1947603510377464

Hong, E., Yik, J., Amanatullah, D. F., Di Cesare, P. E., and Haudenschild, D. R. (2013). c-Maf Transcription Factor Regulates ADAMTS-12 Expression in Human Chondrogenic Cells. Cartilage 4 (2), 177-186. doi:10.1177/ 1947603512472697

Hu, G., Sun, N., Jiang, J., and Chen, X. (2020). Establishment of a 5-gene Risk Model Related to Regulatory T Cells for Predicting Gastric Cancer Prognosis. Cancer Cel. Int. 20, 433. doi:10.1186/s12935-020-01502-6

Jahangir, S., Eglin, D., Potter, N., Khozaei Ravari, M., Stoddart, M. J., Samadikuchaksaraei, A., et al. (2020). Inhibition of Hypertrophy and Improving Chondrocyte Differentiation by MMP-13 Inhibitor Small Molecule Encapsulated in Alginate-Chondroitin Sulfate-Platelet Lysate Hydrogel. Stem Cel. Res. Ther. 11 (1), 436. doi:10.1186/s13287-020-01930-1

Jin, E. J., Park, K. S., Bang, O. S., and Kang, S. S. (2007). Akt Signaling Regulates Actin Organization via Modulation of MMP-2 Activity during Chondrogenesis of Chick Wing Limb Bud Mesenchymal Cells. J. Cel. Biochem. 102 (1), 252-261. doi:10.1002/jcb.21430 
Justen, H. P., Grunewald, E., Totzke, G., Gouni-Berthold, I., Sachinidis, A., Wessinghage, D., et al. (2000). Differential Gene Expression in Synovium of Rheumatoid Arthritis and Osteoarthritis. Mol. Cel. Biol. Res. Commun. 3 (3), 165-172. doi:10.1006/mcbr.2000.0211

Kelwick, R., Desanlis, I., Wheeler, G. N., and Edwards, D. R. (2015). The ADAMTS (A Disintegrin and Metalloproteinase with Thrombospondin Motifs) Family. Genome Biol. 16, 113. doi:10.1186/s13059-015-0676-3

Kevorkian, L., Young, D. A., Darrah, C., Donell, S. T., Shepstone, L., Porter, S., et al. (2004). Expression Profiling of Metalloproteinases and Their Inhibitors in Cartilage. Arthritis Rheum. 50 (1), 131-141. doi:10.1002/art.11433

Kirbas, A., Daglar, K., Gencosmanoglu, G., Yilmaz, Z., Timur, H., Inal, Z., et al. (2016). Total Oxidative and Anti-oxidative Status, and ADAMTS-12 Levels in Placenta Previa and Early-Onset Severe Preeclampsia. Pregnancy Hypertens. 6 (4), 295-299. doi:10.1016/j.preghy.2016.06.001

Koca, G., Gultekin, S. S., Han, U., Kuru, S., Demirel, K., and Korkmaz, M. (2013). The Efficacy of Montelukast as a Protective Agent against 131I-Induced Salivary Gland Damage in Rats: Scintigraphic and Histopathological Findings. Nucl. Med. Commun. 34 (5), 507-517. doi:10.1097/MNM. ob013e32835ffecd

Koike, A., Nishida, N., Inoue, I., Tsuji, S., and Tokunaga, K. (2009). Genome-wide Association Database Developed in the Japanese Integrated Database Project. J. Hum. Genet. 54 (9), 543-546. doi:10.1038/jhg.2009.68

Kullich, W., Fagerer, N., and Schwann, H. (2007). Effect of the NSAID Nimesulide on the Radical Scavenger Glutathione S-Transferase in Patients with Osteoarthritis of the Knee. Curr. Med. Res. Opin. 23 (8), 1981-1986. doi:10. 1185/030079907X223486

Kurz, T., Hoffjan, S., Hayes, M. G., Schneider, D., Nicolae, R., Heinzmann, A., et al. (2006). Fine Mapping and Positional Candidate Studies on Chromosome 5p13 Identify Multiple Asthma Susceptibility Loci. J. Allergy Clin. Immunol. 118 (2), 396-402. doi:10.1016/j.jaci.2006.04.036

Le Maitre, C. L., Pockert, A., Buttle, D. J., Freemont, A. J., and Hoyland, J. A. (2007). Matrix Synthesis and Degradation in Human Intervertebral Disc Degeneration. Biochem. Soc. Trans. 35 (Pt 4), 652-655. doi:10.1042/BST0350652

Lefevre, S., Meier, F. M., Neumann, E., and Muller-Ladner, U. (2015). Role of Synovial Fibroblasts in Rheumatoid Arthritis. Curr. Pharm. Des. 21 (2), 130-141. doi:10.2174/1381612820666140825122036

Li, C., Luo, X., Huang, B., Wang, X., Deng, Y., and Zhong, Z. (2020a). ADAMTS12 Acts as a Cancer Promoter in Colorectal Cancer via Activating the Wnt/betaCatenin Signaling Pathway In Vitro. Ann. Transl Med. 8 (6), 301. doi:10.21037/ atm.2020.02.154

Li, X., Xiao, X., Chang, R., and Zhang, C. (2020b). Comprehensive Bioinformatics Analysis Identifies lncRNA HCG22 as a Migration Inhibitor in Esophageal Squamous Cell Carcinoma. J. Cel Biochem. 121 (1), 468-481. doi:10.1002/jcb. 29218

Liang, L., Zhu, J. H., Chen, G., Qin, X. G., and Chen, J. Q. (2020). Prognostic Values for the mRNA Expression of the ADAMTS Family of Genes in Gastric Cancer. J. Oncol. 2020, 9431560. doi:10.1155/2020/9431560

Lin, E. A., and Liu, C. J. (2009). The Emerging Roles of ADAMTS-7 and ADAMTS12 Matrix Metalloproteinases. Open Access Rheumatol. 1, 121-131. doi:10.2147/ oarrr.s6264

Lin, E. A., and Liu, C. J. (2010). The Role of ADAMTSs in Arthritis. Protein Cell 1 (1), 33-47. doi:10.1007/s13238-010-0002-5

Liu, C. J., Kong, W., Ilalov, K., Yu, S., Xu, K., Prazak, L., et al. (2006a). ADAMTS-7: a Metalloproteinase that Directly Binds to and Degrades Cartilage Oligomeric Matrix Protein. FASEB J. 20 (7), 988-990. doi:10.1096/fj.05-3877fje

Liu, C. J., Kong, W., Xu, K., Luan, Y., Ilalov, K., Sehgal, B., et al. (2006b). ADAMTS12 Associates with and Degrades Cartilage Oligomeric Matrix Protein. J. Biol. Chem. 281 (23), 15800-15808. doi:10.1074/jbc.M513433200

Liu, C. J. (2009). The Role of ADAMTS-7 and ADAMTS-12 in the Pathogenesis of Arthritis. Nat. Clin. Pract. Rheumatol. 5 (1), 38-45. doi:10.1038/ncprheum0961

Llamazares, M., Obaya, A. J., Moncada-Pazos, A., Heljasvaara, R., Espada, J., Lopez-Otin, C., et al. (2007). The ADAMTS12 Metalloproteinase Exhibits Antitumorigenic Properties through Modulation of the Ras-dependent ERK Signalling Pathway. J. Cel Sci. 120 (Pt 20), 3544-3552. doi:10.1242/jcs.005751

Luan, Y., Kong, L., Howell, D. R., Ilalov, K., Fajardo, M., Bai, X. H., et al. (2008). Inhibition of ADAMTS-7 and ADAMTS-12 Degradation of Cartilage Oligomeric Matrix Protein by Alpha-2-Macroglobulin. Osteoarthritis Cartilage. 16 (11), 1413-1420. doi:10.1016/j.joca.2008.03.017
Maass, P. G., Wirth, J., Aydin, A., Rump, A., Stricker, S., Tinschert, S., et al. (2010). A Cis-Regulatory Site Downregulates PTHLH in Translocation $\mathrm{t}(8 ; 12)(\mathrm{q} 13$; p11.2) and Leads to Brachydactyly Type E. Hum. Mol. Genet. 19 (5), 848-860. doi: $10.1093 / \mathrm{hmg} / \mathrm{ddp} 553$

Mani, S., Ghosh, J., Lan, Y., Senapati, S., Ord, T., Sapienza, C., et al. (2019). Epigenetic Changes in Preterm Birth Placenta Suggest a Role for ADAMTS Genes in Spontaneous Preterm Birth. Hum. Mol. Genet. 28 (1), 84-95. doi:10. 1093/hmg/ddy325

Mariani, A., Wang, C., Oberg, A. L., Riska, S. M., Torres, M., Kumka, J., et al. (2019). Genes Associated with Bowel Metastases in Ovarian Cancer. Gynecol, Oncol. 154 (3), 495-504. doi:10.1016/j.ygyno.2019.06.010

Martel-Pelletier, J., Welsch, D. J., and Pelletier, J. P. (2001). Metalloproteases and Inhibitors in Arthritic Diseases. Best Pract. Res. Clin. Rheumatol. 15 (5), 805-829. doi:10.1053/berh.2001.0195

Mead, T. J., McCulloch, D. R., Ho, J. C., Du, Y., Adams, S. M., Birk, D. E., et al. (2018). The Metalloproteinase-Proteoglycans ADAMTS7 and ADAMTS12 Provide an Innate, Tendon-specific Protective Mechanism against Heterotopic Ossification. JCI Insight 3 (7), e92941. doi:10.1172/jci.insight.92941

Miro, X., Meier, S., Dreisow, M. L., Frank, J., Strohmaier, J., Breuer, R., et al. (2012). Studies in Humans and Mice Implicate Neurocan in the Etiology of Mania. Am. J. Psychiatry 169 (9), 982-990. doi:10.1176/appi.ajp.2012.11101585

Moncada-Pazos, A., Obaya, A. J., Fraga, M. F., Viloria, C. G., Capella, G., Gausachs, M., et al. (2009). The ADAMTS12 Metalloprotease Gene Is Epigenetically Silenced in Tumor Cells and Transcriptionally Activated in the Stroma during Progression of Colon Cancer. J. Cel. Sci. 122 (Pt 16), 2906-2913. doi:10.1242/jcs.050468

Moncada-Pazos, A., Obaya, A. J., Llamazares, M., Heljasvaara, R., Suarez, M. F., Colado, E., et al. (2012). ADAMTS-12 Metalloprotease Is Necessary for Normal Inflammatory Response. J. Biol. Chem. 287 (47), 39554-39563. doi:10.1074/jbc. M112.408625

Muhleisen, T. W., Mattheisen, M., Strohmaier, J., Degenhardt, F., Priebe, L., Schultz, C. C., et al. (2012). Association between Schizophrenia and Common Variation in Neurocan (NCAN), a Genetic Risk Factor for Bipolar Disorder. Schizophr Res. 138 (1), 69-73. doi:10.1016/j.schres.2012.03.007

Mysliwiec, M., Panasiuk, B., Debiec-Rychter, M., Iwanowski, P. S., Lebkowska, U., Nowakowska, B., et al. (2015). Co-segregation of Freiberg's infraction with a familial translocation $\mathrm{t}(5 ; 7)(\mathrm{p} 13.3 ; \mathrm{p} 22.2)$ ascertained by a child with cri du chat syndrome and brachydactyly type A1B. Am. J. Med. Genet. A. 167A (2), 445-449. doi:10.1002/ajmg.a.36874

Nah, S. S., Lee, S., Joo, J., Kim, H. K., Sohn, D. R., Kwon, J. T., et al. (2012). Association of ADAMTS12 Polymorphisms with Rheumatoid Arthritis. Mol. Med. Rep. 6 (1), 227-231. doi:10.3892/mmr.2012.867

Namli Kalem, M., Kalem, Z., Yuce, T., and Soylemez, F. (2018). ADAMTS 1, 4, 12, and 13 Levels in Maternal Blood, Cord Blood, and Placenta in Preeclampsia. Hypertens. Pregnancy. 37 (1), 9-17. doi:10.1080/10641955.2017.1397690

Neidhart, M., Hauser, N., Paulsson, M., DiCesare, P. E., Michel, B. A., and Hauselmann, H. J. (1997). Small Fragments of Cartilage Oligomeric Matrix Protein in Synovial Fluid and Serum as Markers for Cartilage Degradation. $\mathrm{Br}$. J. Rheumatol. 36 (11), 1151-1160. doi:10.1093/rheumatology/36.11.1151

Oruc, L., Kapur-Pojskic, L., Ramic, J., Pojskic, N., and Bajrovic, K. (2012). Assessment of Relatedness between Neurocan Gene as Bipolar Disorder Susceptibility Locus and Schizophrenia. Bosn J. Basic Med. Sci. 12 (4), 245-248. doi:10.17305/bjbms.2012.2446

Oztas, E., Ozler, S., Ersoy, A. O., Erkenekli, K., Sucak, A., Ergin, M., et al. (2016). Placental ADAMTS-12 Levels in the Pathogenesis of Preeclampsia and Intrahepatic Cholestasis of Pregnancy. Reprod. Sci. 23 (4), 475-481. doi:10. 1177/1933719115604730

Paulissen, G., El Hour, M., Rocks, N., Gueders, M. M., Bureau, F., Foidart, J. M., et al. (2012). Control of Allergen-Induced Inflammation and Hyperresponsiveness by the Metalloproteinase ADAMTS-12. J. Immunol. 189 (8), 4135-4143. doi:10.4049/jimmunol.1103739

Perez-Garcia, S., Gutierrez-Canas, I., Seoane, I. V., Fernandez, J., Mellado, M., Leceta, J., et al. (2016). Healthy and Osteoarthritic Synovial Fibroblasts Produce a Disintegrin and Metalloproteinase with Thrombospondin Motifs 4, 5, 7, and 12: Induction by IL-1beta and Fibronectin and Contribution to Cartilage Damage. Am. J. Pathol. 186 (9), 2449-2461. doi:10.1016/j.ajpath.2016.05.017

Perez-Garcia, S., Carrion, M., Gutierrez-Canas, I., Villanueva-Romero, R., Castro, D., Martinez, C., et al. (2019a). Profile of Matrix-Remodeling Proteinases in Osteoarthritis: Impact of Fibronectin. Cells 9 (1), 40. doi:10.3390/cells9010040 
Perez-Garcia, S., Carrion, M., Villanueva-Romero, R., Hermida-Gomez, T., Fernandez-Moreno, M., Mellado, M., et al. (2019b). Wnt and RUNX2 Mediate Cartilage Breakdown by Osteoarthritis Synovial Fibroblast-Derived ADAMTS-7 and -12. J. Cel Mol Med. 23 (6), 3974-3983. doi:10.1111/jcmm. 14283

Porter, S., Clark, I. M., Kevorkian, L., and Edwards, D. R. (2005). The ADAMTS Metalloproteinases. Biochem. J. 386 (Pt 1), 15-27. doi:10.1042/BJ20040424

Rabadan, R., Mohamedi, Y., Rubin, U., Chu, T., Alghalith, A. N., Elliott, O., et al. (2020). Identification of Relevant Genetic Alterations in Cancer Using Topological Data Analysis. Nat. Commun. 11 (1), 3808. doi:10.1038/s41467020-17659-7

Rajagopal, K., Ramesh, S., and Madhuri, V. (2020). Early Addition of Parathyroid Hormone-Related Peptide Regulates the Hypertrophic Differentiation of Mesenchymal Stem Cells. Cartilage. doi:10.1177/1947603519894727

Raum, H., Dietsche, B., Nagels, A., Witt, S. H., Rietschel, M., Kircher, T., et al. (2015). A Genome-wide Supported Psychiatric Risk Variant in NCAN Influences Brain Function and Cognitive Performance in Healthy Subjects. Hum. Brain Mapp. 36 (1), 378-390. doi:10.1002/hbm.22635

Rocha, B., Cillero-Pastor, B., Ruiz-Romero, C., Paine, M. R. L., Canete, J. D., Heeren, R. M. A., et al. (2021). Identification of a Distinct Lipidomic Profile in the Osteoarthritic Synovial Membrane by Mass Spectrometry Imaging. Osteoarthritis Cartilage S1063-4584 (21), 00038. doi:10.1016/j.joca.2020. 12.025

Sadic, M., Korkmaz, M., Gultekin, S. S., and Demircan, K. (2016). Alterations in ADAMTS12 Gene Expression in Salivary Glands of Radioiodine-131Administered Rats. Nucl. Med. Commun. 37 (10), 1010-1015. doi:10.1097/ MNM.0000000000000556

Sandy, J. D. (2006). A Contentious Issue Finds Some Clarity: on the Independent and Complementary Roles of Aggrecanase Activity and MMP Activity in Human Joint Aggrecanolysis. Osteoarthritis Cartilage 14 (2), 95-100. doi:10. 1016/j.joca.2005.09.004

Schmidt, S., Arendt, T., Morawski, M., and Sonntag, M. (2020). Neurocan Contributes to Perineuronal Net Development. Neuroscience 442, 69-86. doi:10.1016/j.neuroscience.2020.06.040

Spicer, A. P., Joo, A., and Bowling, R. A., Jr. (2003). A Hyaluronan Binding Link Protein Gene Family Whose Members Are Physically Linked Adjacent to Chondroitin Sulfate Proteoglycan Core Protein Genes: the Missing Links. J. Biol. Chem. 278 (23), 21083-21091. doi:10.1074/jbc.M213100200

Suarez-Carmona, M., Lesage, J., Cataldo, D., and Gilles, C. (2017). EMT and Inflammation: Inseparable Actors of Cancer Progression. Mol. Oncol. 11 (7), 805-823. doi:10.1002/1878-0261.12095

Tauchi, R., Imagama, S., Natori, T., Ohgomori, T., Muramoto, A., Shinjo, R., et al. (2012). The Endogenous Proteoglycan-Degrading Enzyme ADAMTS-4 Promotes Functional Recovery after Spinal Cord Injury. J. Neuroinflammation. 9, 53. doi:10.1186/1742-2094-9-53

van Nie, L., Salinas-Tejedor, L., Dychus, N., Fasbender, F., Hulser, M. L., Cutolo, M., et al. (2020). Dopamine Induces In Vitro Migration of Synovial Fibroblast from Patients with Rheumatoid Arthritis. Sci. Rep. 10 (1), 11928. doi:10.1038/ s41598-020-68836-Z

Wan, R., Hu, J., Zhou, Q., Wang, J., Liu, P., and Wei, Y. (2012). Application of Coexpressed Genes to Articular Cartilage: New Hope for the Treatment of Osteoarthritis (Review). Mol. Med. Rep. 6 (1), 16-18. doi:10.3892/mmr.2012.859

Wang, D., Zhu, T., Zhang, F. B., and He, C. (2011). Expression of ADAMTS12 in Colorectal Cancer-Associated Stroma Prevents Cancer Development and Is a Good Prognostic Indicator of Colorectal Cancer. Dig. Dis. Sci. 56 (11), 3281-3287. doi:10.1007/s10620-011-1723-x

Wang, W., Mandel, J., Bouaziz, J., Commenges, D., Nabirotchkine, S., Chumakov, I., et al. (2015). A Multi-Marker Genetic Association Test Based on the Rasch Model Applied to Alzheimer's Disease. PLoS One 10 (9), e0138223. doi:10.1371/ journal.pone.0138223

Wang, P., Cai, J., Ni, J., Zhang, J., Tang, W., and Zhang, C. (2016). The NCAN Gene: Schizophrenia Susceptibility and Cognitive Dysfunction. Neuropsychiatr. Dis. Treat. 12, 2875-2883. doi:10.2147/NDT.S118160

Wei, J. L., Fu, W., Hettinghouse, A., He, W. J., Lipson, K. E., and Liu, C. J. (2018). Role of ADAMTS-12 in Protecting against Inflammatory Arthritis in Mice by Interacting with and Inactivating Proinflammatory Connective Tissue Growth Factor. Arthritis Rheumatol. 70 (11), 1745-1756. doi:10.1002/art.40552

Witten, A., Ruhle, F., de Witt, M., Barysenka, A., Stach, M., Junker, R., et al. (2020). ADAMTS12, a New Candidate Gene for Pediatric Stroke. PLoS One 15 (8), e0237928. doi:10.1371/journal.pone.0237928

Yu, H., and Zhu, Y. (2012). Expression of ADAMTS-7 and ADAMTS-12 in the Nucleus Pulposus during Degeneration of Rat Caudal Intervetebral Disc. J. Vet. Med. Sci. 74 (1), 9-15. doi:10.1292/jvms.10-0556

Zhang, Q., Huang, M., Wang, X., Xu, X., Ni, M., and Wang, Y. (2012). Negative Effects of ADAMTS-7 and ADAMTS-12 on Endplate Cartilage Differentiation. J. Orthop. Res. 30 (8), 1238-1243. doi:10.1002/jor.22069

Zhang, X., Zheng, P., Li, Z., Gao, S., and Liu, G. (2020). The Somatic Mutation Landscape and RNA Prognostic Markers in Stomach Adenocarcinoma. Onco Targets Ther. 13, 7735-7746. doi:10.2147/OTT.S263733

Zheng, S., Lin, F., Zhang, M., Fu, J., Ge, X., and Mu, N. (2019). AK001058 Promotes the Proliferation and Migration of Colorectal Cancer Cells by Regulating Methylation of ADAMTS12. Am. J. Transl Res. 11 (9), 5869-5878.

Conflict of Interest: The authors declare that the research was conducted in the absence of any commercial or financial relationships that could be construed as a potential conflict of interest.

Copyright (c) 2021 Mohamedi, Fontanil, Cal, Cobo and Obaya. This is an open-access article distributed under the terms of the Creative Commons Attribution License (CC $B Y$ ). The use, distribution or reproduction in other forums is permitted, provided the original author(s) and the copyright owner(s) are credited and that the original publication in this journal is cited, in accordance with accepted academic practice. No use, distribution or reproduction is permitted which does not comply with these terms. 